\title{
A Quantum Walk Model For Emotion Transmission In Serial Reproduction Of Narratives
}

\author{
Jiaqi Huang (huangajq@iu.edu) ${ }^{1}$, Qizi Zhang (zhangqiz@iu.edu) ${ }^{1}$, \\ Jerome Busemeyer (jbusemey@indiana.edu) ${ }^{1}$ \& Fritz Breithaupt (fbreitha@indiana.edu) ${ }^{1}$ \\ ${ }^{1}$ Department of Cognitive Science, Indiana University, 1001 E. 10th Street, Bloomington, IN 47405 USA
}

\begin{abstract}
A quantum walk model is developed for emotion transmission in serial reproduction of narratives. The readers' emotions are represented by density operators, and the influences of the narratives on the readers' emotions are modeled by applying the controlled unitary operators to the density operators. The performance of the quantum model is evaluated on a large corpus of narratives, compared to that of the Bayesian Markov chain model. The quantum model not only outperforms the Bayesian model for all five emotion transmissions presented in the corpus but can also account for order effects in serial reproductions. These results suggest a promising first step towards extending quantum-like models to explain group-level cognition.
\end{abstract}

Keywords: computational modeling; serial reproduction; quantum cognition; emotion transmission;

\section{Introduction}

Numerous empirical findings in cognitive psychology have exhibited anomalies concerning the classical benchmark defined by Kolmogorovian probability axioms and the rules of Boolean logic, triggering a whole new scope of research: the development of models for decision-making based on the quantum formulation of probability theory (Busemeyer \& Bruza, 2012; Haven, Khrennikov, \& Khrennikov, 2013; Khrennikov, Basieva, Dzhafarov, \& Busemeyer, 2014; Pothos $\&$ Busemeyer, 2013, 2022). Although these quantum models provide deep insights into the cognitive anomalies, their scopes are typically confined to the individual level. The next stage of this line of research is to extend quantum models of cognition to the group level. This is very important in understanding anomalies at the group level and how information processing could affect information propagation in social networks and group decision making.

Serial reproduction is an experimental scheme where participants are asked to reproduce a stimulus (e.g. narratives, images), and the reproduced stimulus will then be reproduced by the next participant in a chain. Serial reproduction is chosen as the starting point of developing quantum models in group-level for two reasons: (1) Serial reproduction is an information path of a social network; (2) Large amount of research works, both experimental (Lyons \& Kashima, 2003; Lee, Gelfand, \& Kashima, 2014; Breithaupt, Li, Liddell, Schille-Hudson, \& Whaley, 2018) and theoretical (Xu \& Griffiths, 2010; Hemmer \& Steyvers, 2009; Meylan, Nair, \& Griffiths, 2021), have been done.
In the remainder of this paper, we will first summarize the Bayesian Markov chain model (Xu \& Griffiths, 2010), and present our new quantum model for serial reproduction. We will then compare the performance of the quantum model with the Bayesian model in explaining emotion transmission in a large corpus of serial reproduction of narratives. We finally discuss how the quantum model can account for potential order effects in serial reproduction.

\section{Bayesian model for serial reproduction}

According to Xu \& Griffiths (2010), the outcome of serial reproduction is a sequence of memory reconstructions by the participants in a chain. At step $n+1$, the model assumes that the participant $A_{n+1}$ 's previous experience establishes a prior of the true state of the world $\mu$, with $\mu \sim N\left(\mu_{0}, \sigma_{0}^{2}\right)$, and that the noisy observation $x_{n}$ has a Gaussian distribution with $\mu$ as its center, $x_{n} \mid \mu \sim N\left(\mu, \sigma_{x}^{2}\right)$. The reconstructed true state given the noisy observation $\mu \mid x_{n}$ then follows the Gaussian distribution $N\left(\lambda x_{n}+(1-\lambda) \mu_{0}, \lambda \sigma_{x}^{2}\right)$, where $\lambda=1 /\left(1+\sigma_{x}^{2} / \sigma_{0}^{2}\right)$ (Gelman et al., 2013). Since the reproduced stimulus with attribute values $x_{n+1}$ (e.g. length of a fish in a drawing) only depends on the previous $x_{n}$, the process is a Markov chain with transition probability

$$
p\left(x_{n+1} \mid x_{n}\right)=\int p\left(x_{n+1} \mid \mu\right) p\left(\mu \mid x_{n}\right) d \mu,
$$

where $x_{n+1} \mid \mu \sim N\left(\mu, \sigma_{x}^{2}\right)$. Using the above results, serial reproduction for stimuli with one-dimensional attribute can be seen as a first-order autoregressive process, denoted as AR(1):

$$
x_{n+1}=(1-\lambda) \mu_{0}+\lambda x_{n}+\varepsilon_{n+1}
$$

where $\varepsilon_{n+1} \sim N\left(0, \sigma_{x}^{2}+\sigma_{n}^{2}\right)$ with $\sigma_{n}^{2}=\lambda \sigma_{x}^{2}$.

\section{Quantum model for serial reproduction}

The quantum walk model for serial reproduction is illustrated in Figure 1. The model is developed from a more general quantum walk model for idea propagation in social network (Zhang \& Busemeyer, 2021).

The opinion of the $n$th participant $A_{n}$ conveyed in the stimulus they generate, denoted as $S_{n}$, is modeled by a quantum state $\left|\psi_{n}\right\rangle$ with density operator $\rho_{A_{n}}=\left|\psi_{n}\right\rangle\left|\psi_{n}\right\rangle^{\dagger}$, where $\dagger$ denotes the Hermitian conjugation. The initial opinion state of participant $A_{n+1}$ is modeled by a quantum state $\left|X_{n+1}\right\rangle$ 
with density operator $X_{A_{n+1}}=\left|X_{n+1}\right\rangle\left|X_{n+1}\right\rangle^{\dagger}$. For binary attributes, $\left|\psi_{n}\right\rangle$ and $\left|X_{n+1}\right\rangle$ are two-dimensional states with norm 1 (with respect to the $L^{2}$ norm), and thus can be written as a linear combination of two orthonormal basis choice states $|0\rangle$ and $|1\rangle$, where $|0\rangle$ represents answering "Yes" for the attribute with probability 1 , and $|1\rangle$ represents answering "No" with probability 1 . The above construction could also work for one-dimensional attributes measured by ratings (Ashtiani \& Azgomi, 2015; Martínez-Martínez \& SánchezBurillo, 2016), where $|0\rangle$ now represents the maximum of the rating scale, and $|1\rangle$ represents the minimum. The ratings are then encoded by a linear mapping from the probabilities of $|0\rangle$ and $|1\rangle$. For example, on a rating scale with range [0,5], the state $\frac{1}{\sqrt{2}}|0\rangle+\frac{1}{\sqrt{2}}|1\rangle$ corresponds to a rating of 2.5 .

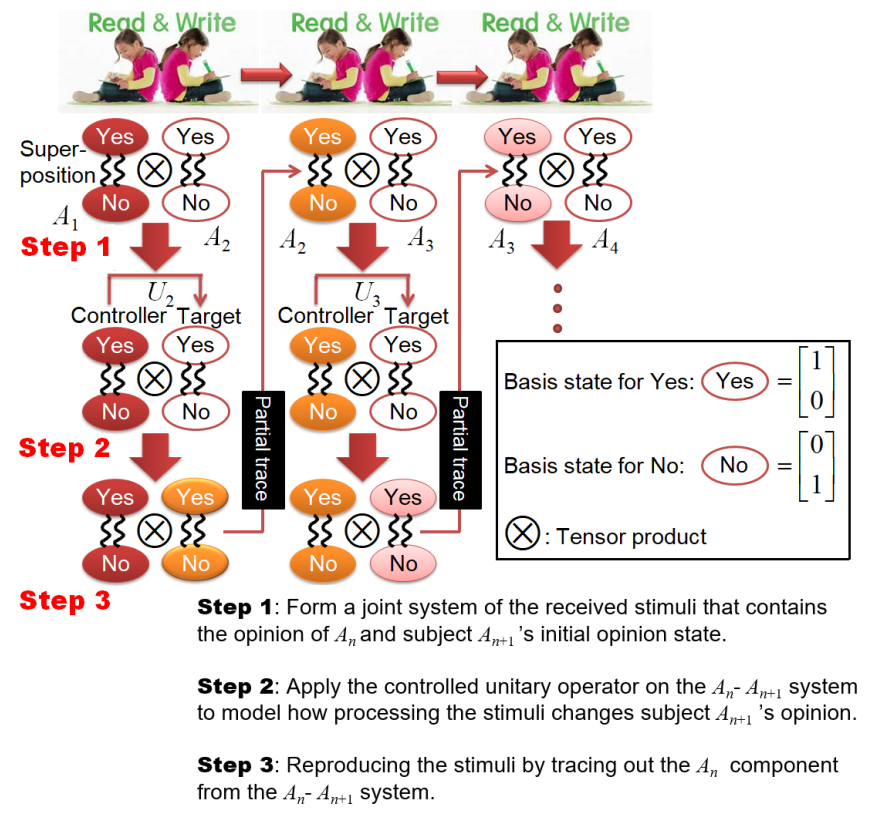

Figure 1: Quantum model for serial reproduction. The opinion state in this example is modeled by a two-dimensional vector containing the probability amplitudes of answering "Yes" or "No" for some attributes of the stimulus. The influence of the opinion of participant $A_{n+1}$ by the opinion of $A_{n}$ expressed in the stimulus $S_{n}$ is modeled by applying a controlled unitary operator $U_{n+1}$ on the $A_{n}-A_{n+1}$ joint system, where the $A_{n}$ part of the joint system represents the controller state, and the $A_{n+1}$ part represents the target state. The opinion state expressed in the reproduced stimulus $S_{n+1}$ is obtained by taking partial trace to trace out the $A_{n}$ component from the joint system, after $A_{n+1}$ has processed $S_{n}$. The reproduced stimulus $S_{n+1}$ will then be received and reproduced by the next participant in the chain.

In step 1, we form a joint system of the opinion of participant $A_{n}$ conveyed in stimulus $S_{n}$, and the initial opinion of $A_{n+1}$ who receives $S_{n}$. The joint system is modeled using a tensor product:

$$
\rho_{n, n+1}=\rho_{A_{n}} \otimes X_{A_{n+1}} .
$$

In step 2, we model how, after processing stimulus $S_{n}$, the opinion of participant $A_{n+1}$ is influenced by $A_{n}$ 's. For one-dimensional attributes, we first define the following controlled unitary operator:

$$
U_{n+1}=M_{0} \otimes R\left(b_{0, n+1} \theta_{0, n}\right)+M_{1} \otimes R\left(b_{1, n+1} \theta_{1, n}\right)
$$

In Equation $4, R(\phi)$ is the two-dimensional rotation operator that models how processing stimulus $S_{n}$ changes the opinion of $A_{n+1}$. The rotation angle $\phi$ for $A_{n+1}$ is determined by the product of the content power of $S_{n}$ regarding choice represented by choice state $|i\rangle$, denoted as $\theta_{i, n}$, and the reading bias of $A_{n+1}$ regarding choice represented by $|i\rangle$, denoted as $b_{i, n+1} . M_{0}$ and $M_{1}$ are projections onto the choice states $|0\rangle$ and $|1\rangle$ correspondingly. The controlled unitary operator is then applied to the joint system operator $\rho_{n, n+1}$ :

$$
\rho_{n+1}=U_{n+1} \cdot \rho_{n, n+1} \cdot U_{n+1}^{\dagger},
$$

where $\rho_{n+1}$ represents the joint system of $A_{n}$ 's opinion conveyed in stimulus $S_{n}$ and $A_{n+1}$ 's opinion after $A_{n+1}$ has processed $S_{n}$. When applying the controlled unitary operator, the $\rho_{A_{n}}$ part of the joint system density operator $\rho_{n, n+1}$ acts as the controller state, and the $X_{A_{n+1}}$ part acts as the target state (see Equation 3). The controller state is not altered by applying $U_{n+1}$, and it controls how the target state $X_{A_{n+1}}$ is changed by $U_{n+1}$. We adopt the control unitary operator, because the opinion of participant $A_{n}$ in stimulus $S_{n}$ can control how $A_{n+1}$ 's initial opinion, encoded in $X_{A_{n+1}}$, is changed after $A_{n+1}$ has processed $S_{n}$, but the opinion in $S_{n}$ is not altered by $A_{n+1}$ processing $S_{n}$.

Finally, in step 3, we model how, after processing $S_{n}$, participant $A_{n+1}$ reproduces stimulus $S_{n+1}$ that conveys their opinion. To do so, we take the partial trace of $\rho_{n+1}$ to trace out the components involving the opinion of $A_{n}$ :

$$
\rho_{A_{n+1}}=\operatorname{Tr}_{A_{n}}\left(\rho_{n+1}\right) \text {. }
$$

The reproduced stimulus $S_{n+1}$ with state $\rho_{A_{n+1}}$ will then be reproduced by $A_{n+2}$ and so on.

In short, there are two theoretical advantages of the quantum walk model over the Bayesian model for serial reproduction. First, the quantum walk model can predict nonlinear relations between $x_{n}$ and $x_{n+1}$, which are the measured values of attributes for $S_{n}$ and $S_{n+1}$ correspondingly. This non-linearity arises naturally from quantum probability theory. Second, the quantum walk model can be easily generalized into a model that explains order effects in serial reproductions. We will discuss these advantages in more detail later.

\section{Emotion transmission in narratives}

Emotion plays a central role in serial reproduction of narratives (Nabi \& Green, 2015; Bilandzic, Kinnebrock, \& Klingler, 2020; Breithaupt, Li, \& Kruschke, 2022). Emotion transmission refers to how the intensity of emotions conveyed in narratives develops during serial reproductions. Previous research (Thompson \& Griffiths, 2021; Stubbersfield, 

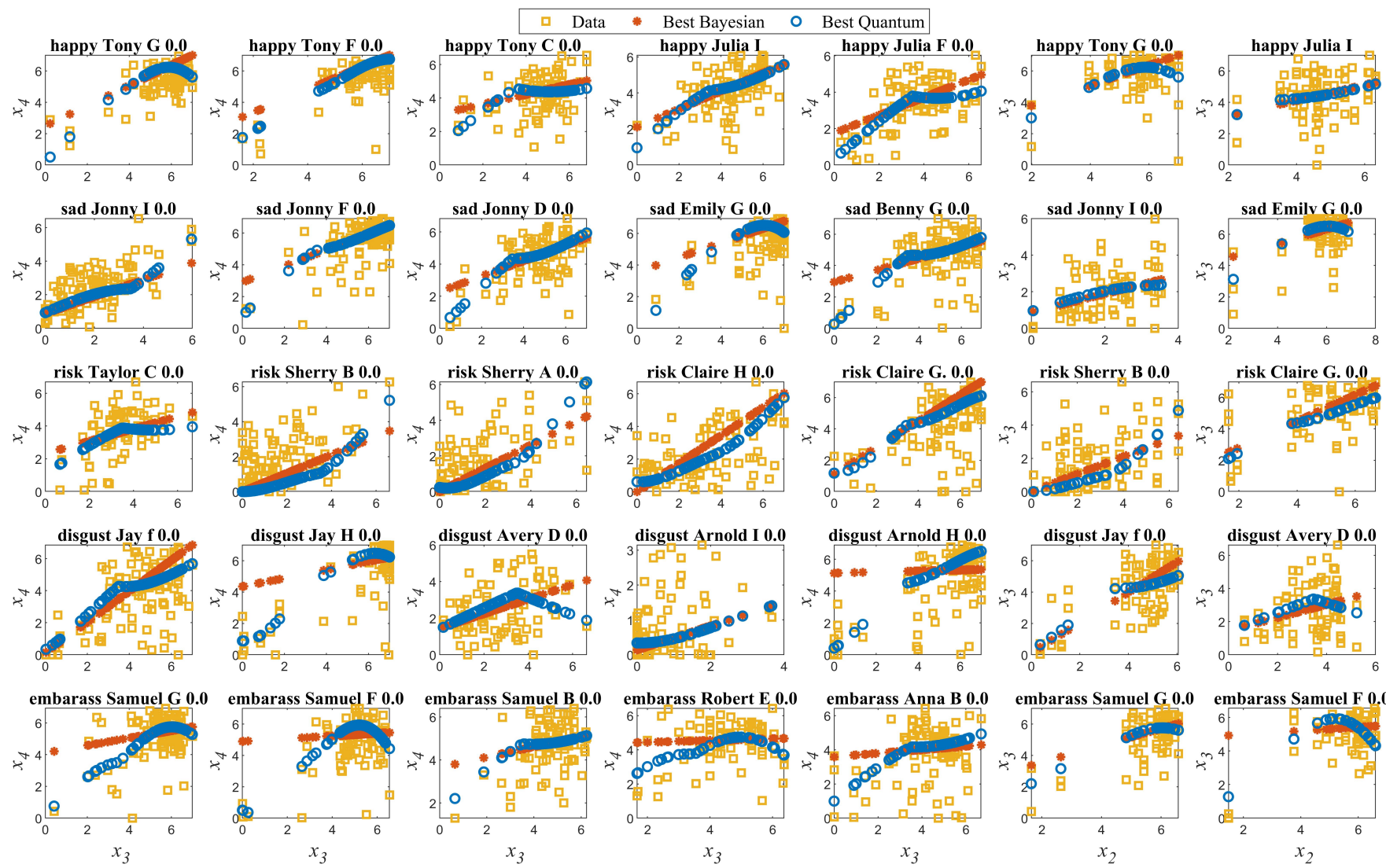

Figure 2: The scatter plots of models' predictions and the data. For all sequences whose heads are the selected original narratives displayed in the titles, the ratings $x_{n+1}$ from the data, the predicted rating of $\hat{x}_{n+1}$ given data $x_{n}$ by the best version of the Bayesian model (in terms of lowest mean BIC for the emotion), and that by the best version of the quantum model, are all plotted against $x_{n}$ from the data. The predictions are computed as the means of the best-fitted normal distributions for the sequences before the distributions are truncated to [0,7]. The first five columns show $x_{4}$ vs $x_{3}$, while the last two show $x_{3}$ vs $x_{2}$, and each row corresponds to one emotion. As observed above, the quantum model can sometimes produce nonlinear predictions of $x_{n+1}$ as a function of data $x_{n}$, while the Bayesian autoregressive model only generates linear predictions. This non-linearity of the quantum model arises from computing quantum probabilities from the complex-valued probability amplitudes.

Tehrani, \& Flynn, 2015; Breithaupt et al., 2022) found that different emotions can transmit very differently and that the transmission, as a function of the steps of the serial reproduction process, is systematic. We consider emotion transmission as the starting point to examine our quantum model for serial reproduction because (1) emotion transmission is important and systematic in narratives; (2) the intensity of emotions is often measured by a one-dimensional rating variable; (3) a recent study (Breithaupt et al., 2022) develops a large corpus (https://osf.io/tpw5e/) about emotion transmission (19,086 retellings; 12,840 participants). The specific dataset examined in this work is a subset of the data in Breithaupt et al., (2022). In this dataset, there are 96 original narratives, denoted as $S_{1}$, and a total number of 8243 sequences developed from these original narratives (different original narratives might be the heads of a different number of sequences). In each sequence, there are three retellings besides the original narrative, denoted as $S_{2}, S_{3}$, and $S_{4}$, and the retelling $S_{n}$ is generated by the participant who reads the pre- vious retelling $S_{n-1}$. For each original narrative, the transmission of one of the five emotions: happiness, sadness, risk, disgust, or embarrassment, is examined. The intensity of emotions is measured using a rating scale from 0 to 7 , with "7" meaning the emotion is strongly presented in the narrative and "0" meaning the emotion is not presented at all (a slider was used that recorded and displayed the ratings to one decimal). The ratings are performed by another group of participants who do not participate in the serial reproduction, and the final rating $x_{n}$ for retelling $S_{n}$ is computed as the mean of all ratings given to $S_{n}$. On average, each first retelling was rated by an average of 5.62 raters, each second retelling by 3.27 raters, and each third retelling by 4.52 .

\section{Bayesian model for emotion transmission}

We develop four versions of Bayesian models: (1) [BS3 $]^{1}$ is the same as the model in Xu \& Griffiths (2010), where a

\footnotetext{
${ }^{1}$ The texts in "[]" are the version names of the models. The reason for naming is explained in the caption of Figure 3.
} 
uniform prior $\mu \sim N\left(\mu_{0}, \sigma_{0}\right)$ and a uniform observation noise $\sigma_{x}^{2}$ are assumed for each participant in the same sequence (for one-dimensional attributes, the model is the AR(1) process shown in Equation 2); (2) [BSA5] is the same as the first version except that we assume a changing observation noise for each participant; (3) [BSB5] is the same as the first version except that rather than a uniform prior, we assume that each participant uses either the reconstruction prior $\mu_{1} \sim N\left(3.5-\phi, \sigma_{0}\right)$ with probability $\omega$, or $\mu_{2} \sim N\left(3.5+\alpha, \sigma_{0}\right)$ with probability $1-\omega$, where $\phi, \alpha \in[0,3.5]$ and $\omega$ are free parameters; (4) [BSAB7] is the same as the third version except that we assume each participant has different observation noises. In practice, since participants are not trained on a specific prior before serial reproduction, the number of reconstruction priors of the participants for this betweenparticipant serial reproduction task could potentially be more than two. However, the main focus of this work is to compare the quantum and the Bayesian models, and thus it is more important to ensure that the quantum model and the Bayesian model share the same assumptions as of the above versions.

\section{Quantum model for emotion transmission}

For emotion transmission, we adopt a special controlled unitary operator:

$$
U_{n+1}= \begin{cases}M_{0} \otimes R\left(\kappa_{n} P_{0, n}+\beta_{n}\right)+M_{1} \otimes I_{2} & P_{0, n} \geq 0.5 \\ M_{0} \otimes I_{2}+M_{1} \otimes R\left(\kappa_{n} P_{0, n}+\beta_{n}\right) & P_{0, n}<0.5\end{cases}
$$

where $P_{0, n} \in[0,1]$ is the $(1,1)$ entry of $\rho_{A_{n}}$ that encodes the probability of the emotion being presented in narrative $S_{n}$, $I_{2}$ is the two-dimensional identity matrix, and $\kappa_{n}$ and $\beta_{n}$ are rotation parameters. The reason for adopting this special unitary is that the unitary operator $U_{n}$ in Equation 4 is designed for narratives with contents of various attributes and readers with different biases to these attributes. When the narratives are of a single one-dimensional attribute, the bias parameter $b_{k, n}$ can be lumped into the content parameter $\theta_{k, n}$.

We develop four versions of quantum models corresponding to each version of the Bayesian model: (1) [Q4] is the four-parameter version of the quantum model which contains an initial opinion state $X=\cos \phi|0\rangle+\sin \phi|1\rangle$ with free parameter $\phi$ that is the same for each participant in the same sequence, a reconstruction noise $\sigma_{0}$ which is constant for each participant, and two rotation parameters $\beta, \kappa$ where $\kappa_{n}=\kappa$ and $\beta_{n}=\beta$ for all $n$; (2) [QA6] is the same as the first version except that, rather than assuming a constant reconstruction noise, we set a separate $\sigma_{n}$ for each participant $A_{n}$; (3) [QB6] is the same as the first version except that participants either use initial opinion state $X=\cos \phi|0\rangle+\sin \phi|1\rangle$ with probability $\omega$, or use $Y=\cos \alpha|0\rangle+\sin \alpha|1\rangle$ with probability $1-\omega$, where $\phi, \alpha$ and $\omega$ are free parameters, and $\cos ^{2} \phi \leq \frac{1}{2}$ and $\cos ^{2} \alpha \geq \frac{1}{2}\left(\cos ^{2} \alpha=\cos ^{2} \phi=\frac{1}{2}\right.$ corresponds to the rating 3.5); (4) [QAB8] is the same as the third version, except that we set a separate $\sigma_{n}$ for each $A_{n}$. We assume that all of $\kappa_{n}, \beta_{n}$ are the same to reduce the fitting complexity. Since the emotion ratings are in the scale [0,7], the predicted $n$th rating by the quantum model is given by

$$
\hat{x}_{n}=7 \times P_{0, n} .
$$

Similarly, the initial state for the $n$th narrative $S_{n}$ is set to be the two dimensional vector,

$$
\left|\psi_{n}\right\rangle=\sqrt{x_{n} / 7}|0\rangle+\sqrt{1-x_{n} / 7}|1\rangle,
$$

where $x_{n}$ is the rating from the data. Finally, the predicted value $\hat{x}_{n}$ will be the mean of a normal distribution with standard deviation $\sigma_{n}$ for computing the likelihood of data $x_{n}$ given the model.

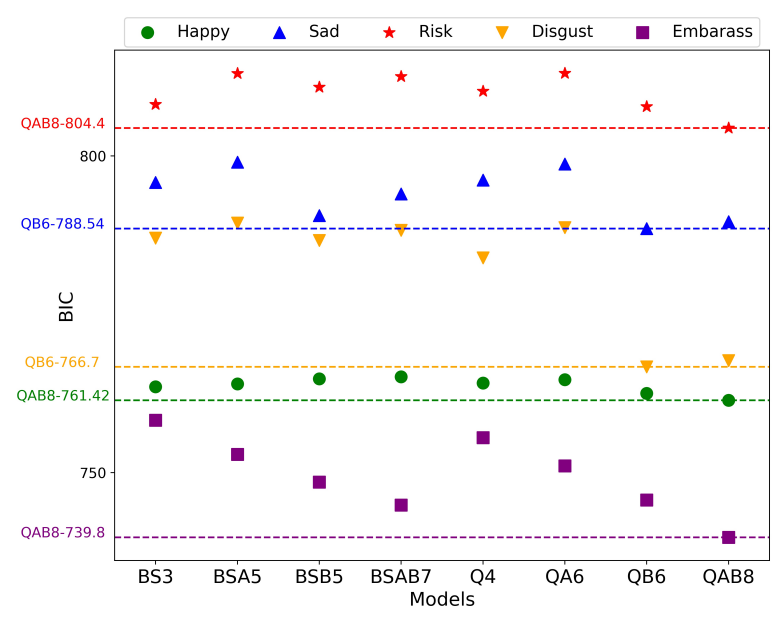

Figure 3: Mean BICs over all initial narratives, for each of the five emotions and each version of the models. The models' names are shown in the $x$ axis, where "BS" stands for Bayesian, and "Q" stands for quantum, "A" stands for versions with a changing observation noise, and "B" stands for versions with two initial beliefs ("AB" means both are presented). The numbers at the end of the names show the number of parameters of the models. The dashed lines represent the lowest BIC scores for the emotion corresponding to the color of the line. The name of the model with the lowest BIC for each emotion and the lowest BIC are labeled in the $y$ axis.

\section{Fitting the models}

We fit one set of parameters for all sequences of retellings with the same original narrative $S_{1}$. By this, we assume that the rating $x_{n+1}$ of any sequence with the same previous narrative $S_{n}$ follows the same probability distribution and that all sequences starting at the same $S_{1}$ transmit emotion in the same way. The models are compared using the Bayesian Information Criterion score (BIC). Since both models use Gaussian likelihood functions while the emotion ratings are in the range [0,7], we truncate the Gaussian functions to [0,7] when computing the BICs. The fitting is performed using the particle swarm algorithm in Matlab global optimization toolbox with a swarm size of 320 and a maximum iteration of 2000. 
The fittings are performed 3 times to check for any convergence to a local minimum since multiple local minimums can exist for the quantum model.

\section{Results and discussions}

Figure 3 shows the results of the BIC fittings. Overall, we found that at least one version of the quantum model provides a better fit of the data compared to any version of the Bayesian model in terms of mean BIC scores over all initial narratives for each emotion.

One reason why the quantum model achieves a better performance is that it can predict a nonlinear relationship between the emotion rating $x_{n}$ of the narrative $S_{n}$ and rating $x_{n+1}$ of $S_{n+1}$, while the AR(1) Bayesian model assumes a linear relationship between $x_{n}$ and $x_{n+1}$. Figure 2 shows how this non-linearity might explain the data better. The nonlinearity here is a natural effect in any quantum probability model, as quantum probability models are based on complexvalued probability amplitudes, whose magnitude squares are the probabilities. In our model, probability amplitudes are converted into probabilities in Equation 5, where both the controlled unitary that encodes the transition probability amplitudes and its Hermitian conjugate are applied to the joint state. And because of this nonlinear conversion from probability amplitudes to probabilities, the model can produce nonlinear predictions even if the controlled unitary operator itself is a linear operator. Except for this part of the model, any other operations including tensor products, partial traces, and the mapping from quantum probabilities to ratings are linear.

Another potential reason could be that for data with a multi-modal distribution, the quantum model could be more sensitive to the multi-modality. As evident in the examples shown in Figure 4, the quantum model can detect some multimodalities that the Bayesian model doesn't detect.
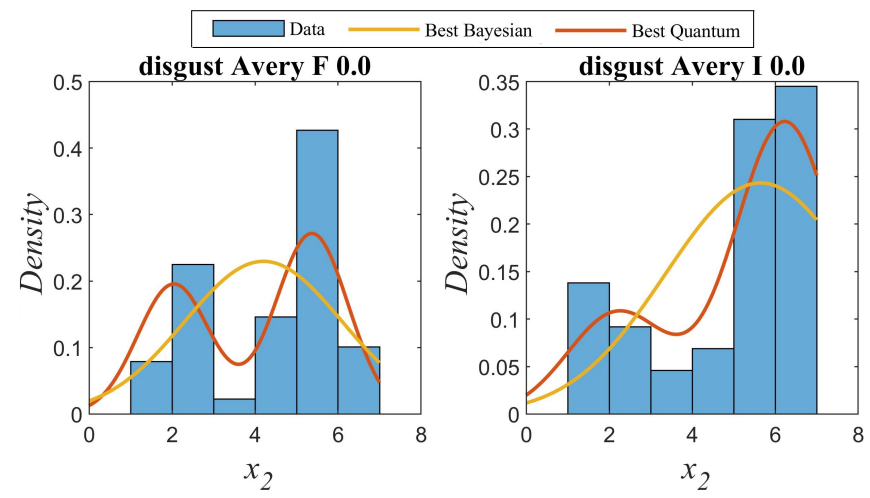

Figure 4: Example original narratives where the quantum model detects a multi-modal distribution and thus produces a better fit, while the Bayesian model does not. The distribution is over the rating $x_{2}$ of all sequences starting from these original narratives. The best-fitted quantum model, in terms of the lowest mean BIC, is of version "QB6", and the bestfitted Bayesian is of version "BSB5".

\section{Order effects in serial reproduction}

In the previous sections, we examine a simple case of serial reproduction: reading and rewriting a narrative where only a one-dimensional attribute (one type of emotion) of the narrative is measured. However, in practice, serial reproduction might also involve making judgments about the received stimulus before or after reproducing the stimulus, and measurement of multiple attributes of the received stimulus. For example, in Lee et al., (2014), participants are first asked to rate both the extent of guilt and the extent of favor of two conflicting parties (strangers and friends) about the received stimulus, and then to reproduce this stimulus. It is a wellknown phenomenon (Hogarth \& Einhorn, 1992; Krosnick \& Alwin, 1987; White, Pothos, \& Busemeyer, 2014) that when multiple judgments are made and multiple attributes are measured, the order of judgments and measurements could affect participants' judgments and opinions. In serial reproduction, since the reproduced stimulus is related to the opinions of the participants, changing the order of tasks and attributes could potentially change the reproduced stimulus. A very important feature of the quantum model is that it can be easily generalized into a model that captures these order effects, and we will briefly discuss examples of two types of order effects that the quantum model accounts for.

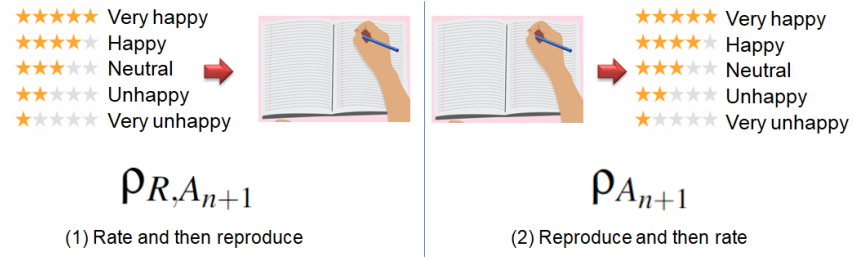

Figure 5: An illustration of the first type of order effect. Participants either first rate how strong an emotion conveyed in the received narrative is, and then reproduce the narrative, or rate after reproducing the narrative. The order effect in the reproduced narrative is a result of the state after processing the previous narrative $\rho_{A_{n+1}}$ collapsing into an eigenstate $\rho_{R, A_{n+1}}$ after rating the emotion.

The first type of order effect is produced by making a choice before/after reproducing a narrative. Consider asking the same participant to both rate how happy the received narrative is, and reproduce the narrative, as illustrated in Figure 5. To explain this order effect, we first need to extend the existing two-dimensional quantum model into a fivedimensional one. The same as in the two-dimensional case, after processing the narrative from $A_{n}$, participant $A_{n+1}$ 's initial state $X_{A_{n+1}}$ is evolved into $\rho_{A_{n+1}}$ through Equation 5 and Equation 6, assuming an extended controlled unitary operator. If a rating of happiness $R$ is made before the narrative reproduction, participant $A_{n+1}$ 's opinion states will collapse into an eigenstate $\rho_{R, A_{n+1}}$ corresponding to the rating $R$. In this case, the reproduced narrative is modeled by the col- 
lapsed state $\rho_{R, A_{n+1}}$. On the other hand, if the ratings are made after reproducing the narrative, the reproduced narrative is modeled by the state $\rho_{A_{n+1}}$. An order effect is then predicted by the quantum model when $\rho_{R, A_{n+1}} \neq \rho_{A_{n+1}}$.

The second type of order effect is produced by a similar experimental paradigm as that of the first, except that instead of asking a single conceptual rating question either before or after writing, we ask two questions about two different attributes of the received narratives, one before and one after rewriting (see Figure 6). For example, let the two attributes be happy and disgusting contents of the received narratives. Measuring the happy content first would potentially guide the participant towards writing a happy story, and vice versa if we were to measure disgusting content first. The quantum model explains this order effect as follows. We first extend the model to five-dimensional, and obtain $\rho_{A_{n+1}}$ using the same stimuli processing procedure as in Equation 5 and Equation 6. Let $\rho_{R_{h}, A_{n+1}}$ and $\rho_{R_{d}, A_{n+1}}$ be the eigenstates $\rho_{A_{n+1}}$ will collapse into after rating happiness as $R_{h}$ and rating disgust as $R_{d}$ respectively. For rating happy content first, the reproduced narrative will have state $\rho_{R_{h}, A_{n+1}}$, and vice versa for rating disgusting content first. When $\rho_{R_{h}, n+1} \neq \rho_{R_{d}, n+1}$, the states of reproduced narratives for the two different orders will therefore be different. Conceptually, this means that the opinion of participant $A_{n+1}$ changes differently when different content is measured first. As a result, the reproduced narratives for the two different orders might convey different intensities of happiness and disgust.

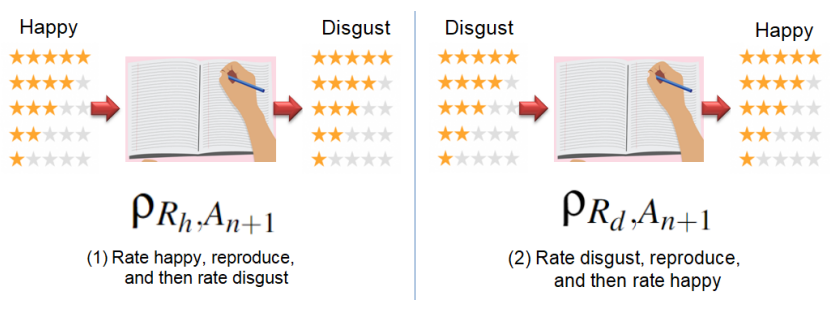

Figure 6: An illustration of the second type of order effect. Participants are asked to rate two attributes about the received narrative (e.g. happy and disgusting contents), and we manipulate the order of measuring these two attributes. The order effect in the reproduced narrative is modeled by different changes to the state of the reproduced narrative in the two different orders.

\section{Conclusions}

We develop a quantum walk model for serial reproduction. The quantum model was compared with a linear Bayesian model on a large dataset about emotion transmission in narratives. The quantum model not only produces a lower mean BIC than the Bayesian model for all five types of emotion transmissions examined but can also account for order effects in serial reproduction. Our results suggest that the quantumlike features of human cognition remain in human communication and affect the idea propagation in group-level cogni- tion.

Future work will focus on different types of order effects in serial reproductions. We will compare the quantum walk model's performance with that of other existing models for order effects in group decision making. Besides, as mentioned, participants may have a wide range of initial beliefs about the strength of an emotion presented in their writings. Thus, a hierarchical model with a distribution of initial beliefs, either quantum or Bayesian, could be developed to better explain the data examined in this work.

\section{Acknowledgments}

This material is based upon work supported by the Air Force Office of Scientific Research under award number FA955020-1-0027.

\section{References}

Ashtiani, M., \& Azgomi, M. A. (2015). A survey of quantumlike approaches to decision making and cognition. Mathematical Social Sciences, 75, 49-80.

Bilandzic, H., Kinnebrock, S., \& Klingler, M. (2020). The emotional effects of science narratives: a theoretical framework. Media and Communication, 8(1), 151-163.

Breithaupt, F., Li, B., \& Kruschke, J. K. (2022). Serial reproduction of narratives preserves emotional appraisals. In Cognition and emotion.

Breithaupt, F., Li, B., Liddell, T. M., Schille-Hudson, E. B., $\&$ Whaley, S. (2018). Fact vs. affect in the telephone game: All levels of surprise are retold with high accuracy, even independently of facts. Frontiers in psychology, 9, 2210.

Busemeyer, J. R., \& Bruza, P. D. (2012). Quantum models of cognition and decision. The Edinburgh Building, Cambridge CB2 8RU, UK: Cambridge University Press.

Gelman, A., Carlin, J. B., Stern, H. S., Dunson, D. B., Vehtari, A., \& Rubin, D. B. (2013). Bayesian data analysis. CRC press.

Haven, E., Khrennikov, A., \& Khrennikov, A. (2013). Quantum social science. The Edinburgh Building, Cambridge CB2 8RU, UK: Cambridge University Press.

Hemmer, P., \& Steyvers, M. (2009). A bayesian account of reconstructive memory. Topics in Cognitive Science, 1(1), 189-202.

Hogarth, R. M., \& Einhorn, H. J. (1992). Order effects in belief updating: The belief-adjustment model. Cognitive psychology, 24(1), 1-55.

Khrennikov, A., Basieva, I., Dzhafarov, E. N., \& Busemeyer, J. R. (2014). Quantum models for psychological measurements: an unsolved problem. PloS one, 9(10), e110909.

Krosnick, J. A., \& Alwin, D. F. (1987). An evaluation of a cognitive theory of response-order effects in survey measurement. Public opinion quarterly, 51(2), 201-219.

Lee, T. L., Gelfand, M. J., \& Kashima, Y. (2014). The serial reproduction of conflict: Third parties escalate conflict through communication biases. Journal of Experimental Social Psychology, 54, 68-72. 
Lyons, A., \& Kashima, Y. (2003). How are stereotypes maintained through communication? the influence of stereotype sharedness. Journal of personality and social psychology, 85(6), 989.

Martínez-Martínez, I., \& Sánchez-Burillo, E. (2016). Quantum stochastic walks on networks for decision-making. Scientific reports, 6(1), 1-13.

Meylan, S. C., Nair, S., \& Griffiths, T. L. (2021). Evaluating models of robust word recognition with serial reproduction. Cognition, 210, 104553.

Nabi, R. L., \& Green, M. C. (2015). The role of a narrative's emotional flow in promoting persuasive outcomes. Media Psychology, 18(2), 137-162.

Pothos, E. M., \& Busemeyer, J. R. (2013). Can quantum probability provide a new direction for cognitive modeling? Behavioral and Brain Sciences, 36(3), 255-274.

Pothos, E. M., \& Busemeyer, J. R. (2022). Quantum cognition. Annual review of psychology, 73.

Stubbersfield, J. M., Tehrani, J. J., \& Flynn, E. G. (2015). Serial killers, spiders and cybersex: Social and survival information bias in the transmission of urban legends. British journal of psychology, 106(2), 288-307.

Thompson, B., \& Griffiths, T. L. (2021). Human biases limit cumulative innovation. Proceedings of the Royal Society B, 288(1946), 20202752.

White, L. C., Pothos, E. M., \& Busemeyer, J. R. (2014). Sometimes it does hurt to ask: The constructive role of articulating impressions. Cognition, 133(1), 48-64.

Xu, J., \& Griffiths, T. L. (2010). A rational analysis of the effects of memory biases on serial reproduction. Cognitive psychology, 60(2), 107-126.

Zhang, Q., \& Busemeyer, J. (2021). A quantum walk model for idea propagation in social network and group decision making. Entropy, 23(5), 622. 